Pacific Journal of Mathematics

PARTIAL ORDERINGS FOR INTEGRAL REPRESENTATIONS
ON CONVEX SETS WITH THE RADON-NIKODÝM PROPERTY 


\title{
PARTIAL ORDERINGS FOR INTEGRAL REPRESENTATIONS ON CONVEX SETS WITH THE RADON-NIKODYM PROPERTY
}

\author{
RICHARD D. BOURGIN
}

\begin{abstract}
The separable extremal ordering and the dilation ordering (as well as equivalent reformulations) have been extensively used in the study of integral representations on closed bounded convex sets with the Radon-Nikodym Property. The relationship between these orderings is clarified and other orderings are introduced.
\end{abstract}

Considerable attention has recently been focused on closed bounded convex sets which have the Radon-Nikodym Property. Such sets exhibit behavior reminiscent in many instances of weakly compact convex sets, and in particular, a theory of integral representations has emerged for these sets which closely parallels that developed by Choquet, Bishop, DeLeeuw and others for compact convex sets. As with the compact case, in all but the most elementary situations (i.e., separable closed bounded convex sets, which correspond in simplicity to metrizable compact convex sets) the techniques center about the use of partial orderings as a means of determining how 'close' to the 'boundary' of the convex set the various measures under consideration live. The exact relationship between two of these orderings, the dilation ordering $<_{d}$ and the separable extremal ordering of Mankiewicz $<_{m}$, is the main subject of this paper.

Each of [17], [4], and [1] is recommended for a comprehensive review of the compact case. Several partial orderings have been used in the noncompact case: Edgar [7] studied the dilation ordering in connection with a general existence theorem, while Bourgin and Edgar [3] used $<_{d}$ and another partial ordering introduced by Edgar in [7], here denoted by $<_{e}$, to prove uniqueness. Independently, St. Raymond [20] employed the Choquet ordering, $<_{c}$, to study the uniqueness question in the separable case. Then Mankiewicz [13] introduced the separable extremal order, and provided a significantly easier proof of the existence of maximal integral representations than had previously been available. Some relationships between these orderings were obtained in [7], [3], [20], [13], and [8], and those needed in this paper are listed in Theorem 1.3.

Section 1 contains background material. The main results, formulated in a variety of ways, are contained in Theorems 2.1, 2.4, 2.5, and Corollary 2.3. Various other partial orderings are suggested by Theorem 2.1 and these are considered in the latter part of $\S 2$. 
[2] contains all the necessary background for this paper.

1. Preliminaries. For any topological space $V$, denote by $\mathscr{B}(V)$ the Borel $\sigma$-field on $V$. (Thus $\mathscr{B}(V)$ is the smallest $\sigma$-algebra of subsets of $V$ which contains the open sets.) Let $(M, d)$ be a complete metric space. Recall that a probability measure $\mu$ on $\mathscr{B}(M)$ is a nonnegative measure with $\mu(M)=1$. Such a measure is tight if, for each $\varepsilon>0$ there is a compact set $D \subset M$ such that $\mu(D)>1-\varepsilon$. The collection of tight probability measures on $\mathscr{B}(M)$ is henceforth written $\mathscr{P}_{t}(M)$. Theorems in [14] and [19] may be combined to show that it is consistent with the Zermelo-Fraenkel axioms of set theory together with the axiom of choice to assume that each probability measure on $M$ is tight. (Tightness is automatic when $M$ is separable. See [16].) Suppose now that $\mathscr{G}$ is a $\sigma$-algebra of subsets of a set $\Gamma$. Then $\mathscr{U}(\mathscr{G})$ refers to the $\mathscr{G}$-universally measurable sets. (Hence $A \in \mathscr{Q}(\mathscr{G})$ if and only if $A$ belongs to the $\mu$-completion of $\mathscr{G}$ for each nonnegative measure $\mu$ on $\mathscr{G}$ such that $\mu(\Gamma)<\infty$.) When $M$ is a complete matric space, $\mathscr{Q}(\mathscr{B}(M))$ will be abbreviated $\mathscr{C}(M)$.

$\mathscr{P}_{t}(M)$ may naturally be embedded in the dual space $C_{b}(M)^{*}$. (Here $C_{b}(M)$ denotes the Banach space of continuous bounded realvalued functions on $M$, equipped with the supremum norm $\|\cdot\|_{\infty}$.) It is understood that when $\mathscr{P}_{t}(M)$ is considered a subset of $C_{b}(M)^{*}$, it has the inherited weak*-topology (so $\mu_{\beta} \rightarrow \mu$ if $\int f d \mu_{\beta} \rightarrow \int f d \mu$ for each $f \in C_{b}(M)$, where $\left.\mu_{\beta}, \mu \in \mathscr{P}_{t}(M) \subset C_{b}(M)^{*}\right)$. A second Banach space which contains $\mathscr{P}_{t}(M)$ in a natural way is $\operatorname{Lip}(M)^{*}$. (For $f \in C_{b}(M)$, let

$$
\|f\|_{L}=\sup \left\{\frac{|f(x)-f(y)|}{d(x, y)}: x \neq y\right\}
$$

and let $\operatorname{Lip}(M)=\left\{f \in C_{b}(M):\|f\|_{L}<\infty\right\}$. When equipped with the norm $\|f\|_{B L}=\|f\|_{\infty}+\|f\|_{L}, \operatorname{Lip}(M)$ is a Banach space.) When $\mathscr{P}_{t}(M)$ is considered a subset of $\operatorname{Lip}(M)^{*}$ the topology on $\mathscr{P}_{t}(M)$ is understood to be the inherited norm topology. That no ambiguity arises from such conventions is a consequence of the following theorems of Dudley [6].

THEOREM 1.1 (Dudley). Let $M$ be a complete metric space. Then $\mathscr{P}_{t}(M) \subset \operatorname{Lip}(M)^{*}$ is a closed bounded convex set. Moreover, the identity map between $\mathscr{P}_{t}(M) \subset \operatorname{Lip}(M)^{*}$ and $\mathscr{P}_{t}(M) \subset C_{b}(M)^{*}$ is a homeomorphism.

If $(\Gamma, \mathscr{G}, Q)$ is a probability space and $C$ is a closed bounded 
convex subset of a Banach space $E$, let $L_{C}^{1}(\Gamma, \mathscr{G}, Q)$, or more briefly $L_{C}^{1}(Q)$, denote the set of Bochner integrable functions on $(\Gamma, \mathscr{G}, Q)$ with $Q$-almost all values in $C$. Then $L_{C}^{1}(Q)$ is a closed bounded convex subset of the Banach space $L_{E}^{1}(Q)$ of $E$-valued Bochner integrable functions. Note that $L_{C}^{1}(Q)$ is separable whenever $C$ is separable and $\mathscr{G}$ is countably generated. For $f \in L_{C}^{1}(Q)$, the probability measure $f(Q)$ on $\mathscr{B}(C)$ is the distribution of $Q$ by $f$ and is defined by the relations:

$$
f(Q)(A)=Q\left(f^{-1}(A)\right) \text { for each } A \in \mathscr{B}(C) .
$$

We are now in a position to state relevant definitions. Throughout this paper $K$ will denote a closed bounded convex subset of a Banach space $E$.

Definition 1.2. $K$ has the Radon-Nikodým Property (RNP) if whenever $(\Gamma, \mathscr{G}, Q)$ is a probability space and $m: \mathscr{G} \rightarrow E$ is an $E$ valued measure whose average range $\{m(A) / Q(A): A \in \mathscr{G}, Q(A)>0\}$ is contained in $K$, then there is an $f \in L_{K}^{1}(Q)$ such that $\int_{A} f d Q=m(A)$ for each $A \in \mathscr{G}$.

DeFinition 1.3. Suppose that $\mu$ and $\nu$ belong to $\mathscr{P}_{t}(K)$.

(a) (Choquet ordering): $\mu<_{c} \nu$ if $\int_{K} f d \mu \leqq \int_{K} f d \nu$ for each convex function $f \in C_{b}(K)$;

(b) (Dilation ordering): $\mu<_{d} \nu$ if there is a function $T \in$ $L_{\mathscr{P}_{t}(K)}^{1}(K, \mathscr{U}(K), \mu)$ (where $\mathscr{P}_{t}(K)$ is here considered a closed bounded convex subset of $\left.\operatorname{Lip}(K)^{*}\right)$ such that

(1) $\int_{K} T d \mu=\nu$, and

(2) for $x \in K$ in a set of $\mu$-measure $1, \int_{K} y d[T(x)](y)=x$. $T$ is then called a $\mu$-dilation. (A reformulation of 2 ) is that for $\mu$-almost every $x$ in $K$, the center of mass, or barycenter, of the measure $T(x)$ is $x$ itself. See [2].)

(c) $\mu<_{e} \nu$ if there are: a probability space $(\Gamma, \mathscr{G}, Q)$, a $\sigma$ algebra $\mathscr{H} \subseteq \mathscr{G}$, and functions $f_{1}$ and $f_{2}$ in $L_{K}^{1}(Q)$ such that

(1) $f_{1}(Q)=\mu$ and $f_{2}(Q)=\nu$; and

(2) $f_{1}=\boldsymbol{E}\left[f_{2} \mid \mathscr{H}\right]$.

(Here $\boldsymbol{E}\left[f_{2} \mid \mathscr{H}\right]$ denotes the Banach-valued conditional expectation of $f_{2}$ given $\mathscr{H}$. See [18] or [2] for details.)

THEOREM 1.3. $<_{c},<_{d}$, and $<_{e}$ are partial orderings on $\mathscr{P}_{t}(K)$. Moreover, for any two measures $\mu, \nu \in \mathscr{P}_{t}(K), \mu<_{c} \nu$ if and only if $\mu<_{d} \nu$, if and only if $\mu<_{e} \nu$.

The results of Theorem 1.3, various portions of which appeared 
originally in [7], [3], and [20], have been generalized to the locally convex case by Edgar [8]. (A proof of the Banach space version of 1.3 appears in [2].)

Let $\alpha$ denote the first uncountable ordinal, and $\Omega$ the uncountable product, $\{0,1\}^{\alpha}$, of two point spaces, equipped with the product topology. For each countable ordinal $\gamma$ and point $\omega \in \Omega$, let $\omega(\gamma)$ be the $\gamma$ th coordinate of $\omega . P$ will denote the product probability measure on $\mathscr{B}(\Omega)$ whose factors assign mass $1 / 2$ to each of $\{0\}$ and $\{1\}$. For $\beta<\alpha$ let $\mathscr{F}_{\beta}$ denote the $P$-completion of the $\sigma$-algebra generated by $\{\{\omega \in \Omega: \omega(\gamma)=0\}: \gamma<\beta\}$. Let $\mathscr{F}=\bigcup_{\beta<\alpha} \mathscr{F}_{\beta}$. In a similar vein, let $\bar{\Omega}=\prod_{i=1}^{\infty}\{0,1\}$ be the countable product of two point spaces. It is a compact metric space when given the product topology. $\bar{P}$ denotes the product probability measure on $\mathscr{B}(\bar{\Omega})$ each of whose factors assigns mass $1 / 2$ to each of $\{0\}$ and $\{1\}$, and $\mathscr{F}$ denotes the $\bar{P}$-completion of $\mathscr{B}(\bar{\Omega})$. In general, a bar over any letter refers to a separable version of the unbarred letter.

Definition 1.4. If $f, g \in L_{K}^{1}(\Omega, \mathscr{F}, P)$, write $f<_{m} g$ if there is a $\beta<\alpha$ such that $f=E\left[g \mid \mathscr{F}_{\beta}\right] . \quad<_{m}$ is called the separable extremal order.

It is easily verified that $<_{m}$ is a partial order. The main link between $L_{K}^{1}(\bar{P})$ and $\mathscr{P}_{t}(K)$ appears below.

THEOREM 1.5. $\left\{f(\bar{P}): f \in L_{K}^{1}(\bar{\Omega}, \overline{\mathscr{F}}, \bar{P})\right\}=\mathscr{P}_{t}(K)$. Moreover the function $f \rightarrow f(\bar{P})$ is continuous from $L_{K}^{1}(\bar{P})$ onto $\mathscr{P}_{t}(K)$.

Proof. The fact that the map $f \rightarrow f(\bar{P})$ is surjective appears in [3, Theorem 3.1], [12], and [2]. Now suppose that $f_{n} \rightarrow f$ in $L_{K}^{1}(\bar{P})$ and that $g \in C_{b}(K)$. Then

$$
\int_{K} g d f_{n}(\bar{P})=\int_{\bar{\Omega}} g \circ f_{n} d \bar{P} \rightarrow \int_{\bar{\Omega}} g \circ f d \bar{P}=\int_{K} g d f(\bar{P}) .
$$

Hence $f_{n}(P) \rightarrow f(P)$ in $\mathscr{P}_{t}(K) \subset C_{b}(K)^{*}$.

The following well-known criterion for Bochner integrability will be used repeatedly in the next section. It is reproduced here for convenient reference. (See, for exmple, [9], [10], or [2].)

THEOREM 1.6. Let $(\Gamma, \mathscr{G}, Q)$ be a probability space and $E$ a Banach space. A function $f: \Gamma \rightarrow E$ is Bochner integrable if and only if (a) there is a set $A \in \mathscr{G}$ with $Q(A)=0$ such that $f(\Gamma \backslash A)$ is a separable subset of $E$ ( $f$ is 'almost separably valued'); (b) for each $F \in E^{*}, F \circ f$ is $\mathscr{G}$-measurable ( $f$ is 'weakly measurable'); (c) $\int\|f\| d Q<\infty$. 
Note that a Bochner integral version of the Fubini theorem is a direct consequence of Theorem 1.6 and the usual Fubini theorem.

2. The separable extremal and dilation orderings. The precise relationship between $<_{d}$ and $<_{m}$ occupies the majority of this section.

Theorem 2.1. Assume that $K$ has the RNP. If $f, g \in L_{K}^{1}(P)$ and $f<_{m} g$ then $f(P)<_{d} g(P)$. Conversely, suppose that $\mu_{1}$ and $\mu_{2}$ are measures in $\mathscr{P}_{t}(K)$. If $\mu_{1}<_{d} \mu_{2}$ there are functions $f$ and $g$ in $L_{K}^{1}(P)$ such that $f(P)=\mu_{1}, g(P)=\mu_{2}$, and $f<_{m} g$.

Proof. If $f, g \in L_{K}^{1}(P)$ and $f<_{m} g$ then there is a countable ordinal $\beta$ such that $f=\boldsymbol{E}\left[g \mid \mathscr{F}_{\beta}\right]$. For any convex $F \in C_{b}(K)$ it follows from Jensen's inequality [15] that

$$
\begin{aligned}
\int_{K} F d f(P) & =\int_{\Omega} F \circ f d P=\int_{\Omega} F \circ E\left[g \mid \mathscr{F}_{\beta}\right] d P \\
& \leqq \int_{\Omega} E\left[F \circ g \mid \mathscr{F}_{\beta}\right] d P=\int_{\Omega} F \circ g d P=\int_{\Omega} F d g(P)
\end{aligned}
$$

whence $f(P)<_{c} g(P)$. The fact that $f(P)<_{d} g(P)$ is a consequence of Theorem 1.3.

Suppose now that $\mu_{1}, \mu_{2} \in \mathscr{P}_{t}(K)$ and that $\mu_{1}<_{d} \mu_{2}$. Since $\mu_{1}$ and $\mu_{2}$ are tight they have separable support. Let $\bar{K}$ be the intersection with $K$ of the closed linear span of the support of $\mu_{1}+\mu_{2}$. Then $\bar{K}$ is a separable closed bounded convex set. Pick any $\bar{f} \in L_{\bar{K}}^{1}(\bar{P})$ for which $\bar{f}(\bar{P})=\mu_{1}\left(\right.$ cf. Theorem 1.5) and let $T: \bar{K} \rightarrow \mathscr{P}_{t}(\bar{K})$ be a $\mu_{1}$-dilation such that $\int_{\vec{K}} T d \mu_{1}=\mu_{2}$. By modifying $T$ on a set of $\mu_{1}$-measure 0 we may also assume that $T(x)$ has barycenter $x$ for each $x \in \bar{K}$. (Let $\varepsilon_{x}$ denote the Dirac measure, or point mass, at $x$. Then $x \rightarrow \varepsilon_{x}$ is continuous. On a $\mathscr{U}(\bar{K})$ set of measure 0 containing the points $x$ at which $T(x)$ fails to have barycenter $x$, replace $T(x)$ by $\varepsilon_{x}$. In this way the modified $T$ is still $\mathscr{C}(\bar{K})-\mathscr{P}\left(\mathscr{P}_{t}(\bar{K})\right)$-measurable.) Observe that $T(\bar{f}(\bar{\omega})) \in \mathscr{P}_{t}(\bar{K})$ for each $\bar{\omega} \in \bar{\Omega}$. It follows from Theorem 1.5 that, for a given $\bar{\omega} \in \bar{\Omega}$, there is at least one $\bar{h} \in L_{\bar{K}}^{1}(\bar{P})$ such that $\bar{h}(\bar{P})=$ $T(\bar{f}(\overline{\boldsymbol{\omega}}))$. It is critical in what follows that the correspondence $\bar{\omega} \rightarrow \bar{h}$ be made in a measurable fashion, and this may be accomplished as follows. The map which takes $\bar{g}$ to $\bar{g}(\bar{P})$ from $L_{\bar{K}}^{1}(\bar{P})$ onto $\mathscr{P}_{t}(\bar{K})$ is both continuous and surjective by Theorem 1.5. Moreover, $L_{K}^{1}(\bar{P})$ and $\mathscr{P}_{t}(\bar{K})$ are completely metrizable and separable since $\bar{K}$ is separable (cf. Theorem 1.1). A special case of the Kuratowski-RyllNardzewski selection theorem ([11] or [5]) asserts the existence of a function $S: \mathscr{P}_{t}(\bar{K}) \rightarrow L_{\bar{K}}^{1}(\bar{P})$ which is $\mathscr{Q}\left(\mathscr{P}_{t}(\bar{K})\right)-\mathscr{B}\left(L_{\bar{K}}(\bar{P})\right)$-measurable such that $[S(\nu)](\bar{P})=\nu$ for each $\nu \in \mathscr{P}_{t}(\bar{K})$. In order to examine the map $S \circ T \circ \bar{f}: \bar{\Omega} \rightarrow L_{K}^{1}(\bar{P})$, the following easily established facts 
will be used (see, for example [2]).

Suppose that $\left(\Gamma_{1}, \mathscr{G}_{1}\right)$ and $\left(\Gamma_{2}, \mathscr{G}_{2}\right)$ are measurable spaces.

(a) If $H: \Gamma_{1} \rightarrow \Gamma_{2}$ is $\mathscr{G}_{1}-\mathscr{G}_{2}$-measurable, it is also $\mathscr{U}\left(\mathscr{G}_{1}\right)-$ $\mathscr{U}\left(\mathscr{G}_{2}\right)$ measurable;

(b) $\mathscr{U}\left(\mathscr{U}\left(\mathscr{G}_{1}\right)\right)=\mathscr{U}\left(\mathscr{G}_{1}\right)$;

(c) If $\nu$ is a nonnegative measure on $\mathscr{G}_{1}$ with $\nu\left(\Gamma_{1}\right)<\infty$ and if the $\nu$-completion of $\mathscr{G}_{1}$ is again $\mathscr{G}_{1}$ then $\mathscr{\mathscr { L }}\left(\mathscr{G}_{1}\right)=\mathscr{G}_{1}$.

If $U \subset L_{\bar{K}}^{1}(\bar{P})$ is (relatively) open then $S^{-1}(U) \in \mathscr{K}\left(\mathscr{P}_{t}(\bar{K})\right)$. Since $T \in L_{\mathscr{S}_{t}(\bar{K})}^{1}\left(\bar{K}, \mathscr{U}(\bar{K}), \mu_{1}\right), T$ is $\mathscr{U}(\bar{K})-\mathscr{B}\left(\mathscr{P}_{t}(\bar{K})\right)$-measurable hence $T$ is $\mathscr{U}(\bar{K})-\mathscr{U}\left(\mathscr{P}_{t}(\bar{K})\right)$-measurable by (a) and (b) above. It follows that $T^{-1}\left(S^{-1}(U)\right) \in \mathscr{U}(\bar{K})$. But $\bar{f} \in L_{\bar{K}}^{1}(\bar{P})$ whence $\bar{f}$ is $\overline{\mathscr{F}}-\mathscr{B}(\bar{K})$ measurable. Use (a) and (c) above to show that $\bar{f}$ is $\overline{\mathscr{F}}-\mathscr{U}(\bar{K})$ measurable. It follows that $(S \circ T \circ \bar{f})^{-1}(U) \in \overline{\mathscr{F}}$. Since $\bar{K}$ is separable and bounded it follows from Theorem 1.6 that $S \circ T \circ \bar{f}: \bar{\Omega} \rightarrow L_{\bar{K}}^{1}(\bar{P})$ is Bochner integrable. That is, $S \circ T \circ \bar{f} \in L_{L_{K}^{1}(\bar{P})}^{1}(\bar{P})$.

Define $\bar{g}_{1}: \bar{\Omega} \times \bar{\Omega} \rightarrow \bar{K}$ as follows:

$$
\bar{g}_{1}\left(\bar{\omega}, \bar{\omega}^{\prime}\right)=[S \circ T \circ \bar{f}(\bar{\omega})]\left(\bar{\omega}^{\prime}\right) .
$$

In Proposition 2.2 below it is established that there is a function $\bar{g}: \bar{\Omega} \times \bar{\Omega} \rightarrow \bar{K}$ such that $\bar{g}(\bar{\omega}, \cdot)=\bar{g}_{1}(\bar{\omega}, \cdot)$ [a.e. $\bar{P}$ ] for each $\bar{\omega} \in \bar{\Omega}$, and $\bar{g}$ is $\overline{\mathscr{F}} \times \overline{\mathscr{F}}-\mathscr{B}(\bar{K})$-measurable. Temporarily assume that such a $\bar{g}$ has already been constructed. We will have occasion to factor $\bar{\Omega}$ out of $\Omega$ and write $\Omega=\bar{\Omega} \times \Omega_{1}$, and even $\Omega=\bar{\Omega} \times \bar{\Omega} \times \Omega_{2}$. The probability measure $P$ will similarly be factored as $P=\bar{P} \times P_{1}$ and $P=\bar{P} \times \bar{P} \times P_{2}$. Functions on $\bar{\Omega}$ will be identified with functions on $\Omega=\bar{\Omega} \times \Omega_{1}$ which depend only on the $\bar{\Omega}$ coordinates (so that $\bar{f}: \bar{\Omega} \rightarrow E$, for example, corresponds to $f: \Omega=\bar{\Omega} \times \Omega_{1} \rightarrow E$ given by $\left.f\left(\bar{\omega}, \omega_{1}\right)=\bar{f}(\bar{\omega})\right)$. Functions on $\bar{\Omega} \times \bar{\Omega}$ may similarly be identified with functions on $\Omega=\bar{\Omega} \times \bar{\Omega} \times \Omega_{2}$ and in particular, $\bar{g}: \bar{\Omega} \times \bar{\Omega} \rightarrow \bar{K}$ will be identified with $g: \Omega \rightarrow \bar{K}$. We first show that $\boldsymbol{E}\left[g \mid \mathscr{F}_{\xi}\right]=f$ where $\xi$ is the first infinite ordinal. Certainly $f$ is $\mathscr{F}_{\xi}$-measurable. If, now, $A \in \mathscr{F}_{\xi}$, there is a set $B \in \mathscr{B}(\bar{\Omega})$ such that the symmetric difference of $A$ and $B \times \Omega_{1}$ is of $P$-measure 0 . Consequently

$$
\begin{aligned}
\int_{A} g d P & =\int_{B \times \Omega_{1}} g d P \\
& =\int_{B} \int_{\bar{\Omega}} \int_{\Omega_{2}} g\left(\bar{\omega}, \bar{\omega}^{\prime}, \omega_{2}\right) d P_{2}\left(\omega_{2}\right) d \bar{P}\left(\bar{\omega}^{\prime}\right) d \bar{P}(\bar{\omega}) \\
& =\int_{B} \int_{\bar{\Omega}} \bar{g}\left(\bar{\omega}, \bar{\omega}^{\prime}\right) d \bar{P}\left(\bar{\omega}^{\prime}\right) d \bar{P}(\bar{\omega})=\int_{B} \int_{\bar{\Omega}} \bar{g}_{1}\left(\bar{\omega}, \bar{\omega}^{\prime}\right) d \bar{P}\left(\bar{\omega}^{\prime}\right) d \bar{P}(\bar{\omega}) \\
& =\int_{B} \int_{\bar{\Omega}}[S \circ T \circ \bar{f}(\bar{\omega})]\left(\bar{\omega}^{\prime}\right) d \bar{P}\left(\bar{\omega}^{\prime}\right) d \bar{P}(\bar{\omega}) \\
& =\int_{B} \int_{\bar{K}} x d[S \circ T \circ \bar{f}(\bar{\omega})](\bar{P})(x) d \bar{P}(\bar{\omega})
\end{aligned}
$$




$$
\begin{aligned}
= & \int_{B} \int_{\bar{K}} x d[T(\bar{f}(\overline{\boldsymbol{\omega}}))](x) d \bar{P}(\overline{\boldsymbol{\omega}})=\int_{B} \bar{f}(\overline{\boldsymbol{\omega}}) d \bar{P}(\overline{\boldsymbol{\omega}}) \\
& \int_{B} \int_{\Omega_{1}} f\left(\overline{\boldsymbol{\omega}}, \omega_{1}\right) d P_{1}\left(\omega_{1}\right) d \bar{P}(\overline{\boldsymbol{\omega}})=\int_{B \times \Omega_{1}} f d P=\int_{A} f d P .
\end{aligned}
$$

Hence $\boldsymbol{E}\left[g \mid \mathscr{F}_{\xi}\right]=f$.

The next calculation establishes that $\bar{g}(\bar{P} \times \bar{P})=\mu_{2}$ (or equivalently, $\left.g(P)=g\left(\bar{P} \times \bar{P} \times P_{2}\right)=\mu_{2}\right)$. Note first that for any $\mu \in \mathscr{P}_{t}(\bar{K})$ we have $\mu=\int_{\bar{K}} \varepsilon_{x} d \mu(x)$. Now

$$
\begin{aligned}
\mu_{2} & =\int_{\bar{K}} T(x) d \mu_{1}(x)=\int_{\bar{K}} T(x) d \bar{f}(\bar{P})(x) \\
& =\int_{\bar{\Omega}} T \circ \bar{f}(\bar{\omega}) d \bar{P}(\bar{\omega})=\int_{\overline{\bar{\alpha}}}[S \circ T \circ \bar{f}(\bar{\omega})](\bar{P}) d \bar{P}(\bar{\omega}) \\
& =\int_{\bar{\Omega}} \int_{\bar{\Omega}} \varepsilon_{\left[S^{\circ} T^{\circ} \bar{f}(\bar{\omega})\right]\left(\bar{\omega}^{\prime}\right)} d \bar{P}\left(\bar{\omega}^{\prime}\right) d \bar{P}(\bar{\omega}) . \\
& =\int_{\bar{\Omega}} \int_{\bar{\Omega}} \varepsilon_{\bar{g}_{1}\left(\bar{\omega}, \bar{\omega}^{\prime}\right)} d \bar{P}\left(\bar{\omega}^{\prime}\right) d \bar{P}(\bar{\omega}) \\
& =\int_{\bar{\Omega} \times \bar{\Omega}} \varepsilon_{\bar{g}\left(\bar{\omega}, \bar{\omega}^{\prime}\right)} d \bar{P} \times \bar{P}\left(\bar{\omega}^{\prime}, \bar{\omega}\right) \\
& =\int_{\bar{K}} \varepsilon_{x} d \bar{g}(\bar{P} \times \bar{P})(x)=\int_{\bar{K}} \varepsilon_{x} d g(P)(x)=g(P) .
\end{aligned}
$$

Hence $g(P)=\mu_{2}, f(P)=\mu_{1}$, and $\boldsymbol{E}\left[g \mid \mathscr{F}_{\xi}\right]=f$ so that $f<_{m} g$. The proof of Theorem 2.1 thus rests on the existence of $\bar{g}$ as asserted in the previous paragraphs.

Recall that a probability space $(\Gamma, \mathscr{G}, Q)$ is said to be separable if there is a countable collection $B_{1}, B_{2}, \cdots$ of sets in $\mathscr{G}$ such that whenever $A \in \mathscr{G}$ there is a $B$ in the $\sigma$-algebra generated by $\left\{B_{1}, B_{2}, \cdots\right\}$ such that $Q(A \triangle B)=0 . \quad(\triangle$ denotes symmetric difference.) I wish to thank Robert Cogburn for useful discussions concerning the next result.

Proposition 2.2. Let $(\Gamma, \mathscr{G}, Q)$ be a separable probability space, and suppose that $\bar{K}$ is a separable closed bounded convex subset of a Banach space $E$. Assume further that $\bar{K}$ has the RNP. If $g: \Gamma \rightarrow L_{K}^{1}(Q)$ is Bochner integrable (i.e., $\left.g \in L_{L_{K}^{1}(Q)}^{1}(Q)\right)$ then there is a function $h: \Gamma \rightarrow L_{\bar{K}}^{1}(Q)$ such that

(a) $g(\omega, \cdot)=h(\omega, \cdot)[$ a.e. Q] holds for each choice of $\omega \in \Gamma$ (where $g\left(\omega, \omega^{\prime}\right)$ is alternate notation for $[g(\omega)]\left(\omega^{\prime}\right)$, and similarly for $\left.h\left(\omega, \omega^{\prime}\right)\right)$;

(b) $h: \Gamma \times \Gamma \rightarrow \bar{K}$ is $\mathscr{G} \times \mathscr{G}$-measurable.

Moreover, functions $h$ can be constructed satisfying (a) above but not (b). 
Proof. Without loss of generality assume $0 \in \bar{K}$. Let $B_{1}, B_{2}, \cdots$ be sets in $\mathscr{G}$ as in the definition of separability of $(\Gamma, \mathscr{G}, Q)$ before the statement of the proposition, and for each $n$ let $\mathscr{G}_{n}$ be the $\sigma$ algebra generated by $\left\{B_{1}, \cdots, B_{n}\right\}$. Thus $\mathscr{G}_{n}$ consists of arbitrary unions of minimal nonempty intersections of various of the $B_{i}$ 's, $i=1, \cdots, n$. These minimal nonempty intersections are the atoms of $\mathscr{G}_{n}$, and for each $n$ and each $\omega \in \Gamma$, there is a unique atom of $\mathscr{G}_{n}$, henceforth denoted by $A_{n}(\omega)$, which contains $\omega$.

For each $\omega \in \Gamma$ and $A \in \mathscr{G}$ let

$$
P(\omega, A)=\int_{A}[g(\omega)]\left(\omega^{\prime}\right) d Q\left(\omega^{\prime}\right) .
$$

$(P(\cdot, \cdot)$ may be thought of as an $E$-valued transition probability function.) Note that for each $\omega \in \Gamma, P(\omega, \cdot)$ is an $E$-valued measure absolutely continuous with respect to $Q$, whose average range belongs to $\bar{K}$. For each positive integer $n$ let

$$
h_{n}\left(\omega, \omega^{\prime}\right)=\left\{\begin{array}{ccc}
\frac{P\left(\omega, A_{n}\left(\omega^{\prime}\right)\right)}{Q\left(A_{n}\left(\omega^{\prime}\right)\right)} & \text { if } & Q\left(A_{n}\left(\omega^{\prime}\right)\right) \neq 0 \\
0 & \text { if } & Q\left(A_{n}\left(\omega^{\prime}\right)\right)=0
\end{array} .\right.
$$

We first show that $h_{n}: \Gamma \times \Gamma \rightarrow E$ is $\mathscr{G} \times \mathscr{G}$-measurable. A straightforward reduction shows that $h_{n}$ is $\mathscr{G} \times \mathscr{G}$-measurable if, for each atom $C$ of $\mathscr{G}_{n}$ for which $Q(C)>0$, the map $G_{C}: \Gamma \rightarrow E$ defined by

$$
G_{C}(\omega)=\int_{C} g\left(\omega, \omega^{\prime}\right) d Q\left(\omega^{\prime}\right)
$$

is $\mathscr{G}$-measurable. Observe that $G_{C}$ is $\bar{K}$-valued (since $0 \in \bar{K}$ ). Since $G_{C}$ has separable range ( $\bar{K}$ is separable) it suffices to show that $G_{C}$ is weakly measurable (cf. Theorem 1.6). That is, if $F \in E^{*}$ then it must be demonstrated that $F \circ G_{C}: \Omega \rightarrow R$ is $\mathscr{G}$-measurable. In this regard define $H_{F} \in L_{E}^{1}(Q) *$ by

$$
H_{F}(f)=\int_{C} F \circ f(\omega) d Q(\omega) \text { for } f \in L_{E}^{1}(Q) .
$$

Since $g \in L_{L_{\bar{K}}(Q)}^{1}(Q)$, it is weakly measurable, and in particular for $H_{F}$ as above, $H_{F} \circ g$ is $\mathscr{G}$-measurable. But $H_{F} \circ g=F \circ G_{C}$ so that $F \circ G_{C}$ is indeed $\mathscr{G}$-measurable. Consequently $h_{n}$ is $\mathscr{G} \times \mathscr{G}$-measurable.

Next we claim that, for fixed $\omega \in \Gamma,\left(h_{n}(\omega, \cdot), \mathscr{G}_{n}, Q\right)_{n=1}^{\infty}$ is a $\bar{K}$ valued martingale. (Indeed, if $C$ is an atom of $\mathscr{G}_{n}$ with $Q(C)>0$, and if the atoms of $\mathscr{G}_{n+1}$ of positive $Q$-measure contained in $C$ are $C_{1}, \cdots, C_{k}$ then $Q\left(C \backslash \bigcup_{i=1}^{k} C_{i}\right)=0$ and 


$$
\begin{aligned}
\int_{C} h_{n}\left(\omega, \omega^{\prime}\right) d Q\left(\omega^{\prime}\right) & =P(\omega, C)=\sum_{i=1}^{k} P\left(\omega, C_{i}\right) \\
& =\sum_{i=1}^{k} \int_{C_{i}} h_{n+1}\left(\omega, \omega^{\prime}\right) d Q\left(\omega^{\prime}\right) \\
& =\int_{C} h_{n+1}\left(\omega, \omega^{\prime}\right) d Q\left(\omega^{\prime}\right) .
\end{aligned}
$$

The martingale conclusion follows easily from this calculation.) Since $\bar{K}$ has the RNP, for each $\omega \in \Gamma$ we have

(*) $\quad \lim _{n \rightarrow \infty} h_{n}\left(\omega, \omega^{\prime}\right)$ exists for $Q$-almost all $\omega^{\prime}$.

Let $A=\left\{\left(\omega, \omega^{\prime}\right): \lim _{n \rightarrow \infty} h_{n}\left(\omega, \omega^{\prime}\right)\right.$ exists $\}$ and let that limit be denoted by $h\left(\omega, \omega^{\prime}\right)$ for $\left(\omega, \omega^{\prime}\right) \in A$. Note that $A \in \mathscr{G} \times \mathscr{G}$ since each $h_{n}$ is jointly measurable. It follows from $(*)$ that $\chi_{A}$, the characteristic function of $A$, has the property that

$$
\int_{\Gamma \times \Gamma} \chi_{A} d Q \times Q=\int_{\Gamma} \int_{\Gamma} \chi_{A}\left(\omega, \omega^{\prime}\right) d Q\left(\omega^{\prime}\right) d Q(\omega)=\int_{\Gamma} 1 d Q(\omega)=1 .
$$

Consequently $h$ is defined [a.e. $Q \times Q$ ], and it is jointly measurable.

In order to prove that $h(\omega, \cdot)=g(\omega, \cdot)$ [a.e. Q] for each $\omega \in \Gamma$, suppose initially that $C$ is an atom of $\mathscr{G}_{n}$ for some $n$. Then

$$
\begin{aligned}
\int_{C} h\left(\omega, \omega^{\prime}\right) d Q\left(\omega^{\prime}\right) & =\int_{C} h_{n}\left(\omega, \omega^{\prime}\right) d Q\left(\omega^{\prime}\right) \\
& =P(\omega, C)=\int_{C} g\left(\omega, \omega^{\prime}\right) d Q\left(\omega^{\prime}\right) .
\end{aligned}
$$

It follows that $\int_{D} h\left(\omega, \omega^{\prime}\right) d Q\left(\omega^{\prime}\right)=\int_{D} g\left(\omega, \omega^{\prime}\right) d Q\left(\omega^{\prime}\right)$ for $D \in \bigcup_{n=1}^{\infty} \mathscr{G}_{n}$. But for each $\varepsilon>0$ and $B \in \mathscr{G}$ there is a set $D \in \bigcup_{n=1}^{\infty} \mathscr{G}_{n}$ such that $Q(B \triangle D)<\varepsilon$. Since $\bar{K}$ is bounded (and since the ranges of $h$ and $g$ are [a.e. $Q]$ in $\bar{K}$ ) it is straightforward to check that

$$
\int_{B} h\left(\omega, \omega^{\prime}\right) d Q\left(\omega^{\prime}\right)=\int_{B} g\left(\omega, \omega^{\prime}\right) d Q\left(\omega^{\prime}\right)
$$

for each $B \in \mathscr{G}$. Thus $g(\omega, \cdot)=h(\omega, \cdot)$ [a.e. Q] as was to be shown.

In order to produce an example of a function $h_{1}$ which satisfies (a) in the statement of the proposition but not (b), let $\Gamma=\bar{K}=[0,1]$, $\mathscr{G}=\mathscr{B}([0,1])$, and let $\lambda$ denote Lebesgue measure on $\mathscr{G}$. Let $f: \Gamma \rightarrow \Gamma$ be a function whose graph (as a subset $[0,1] \times[0,1]$ ) is nonmeasurable. (See, for example, B. R. Gelbaum and J. M. H. Olmstead, Counterexamples in Analysis, Holden Day, Inc., San Francisco, Example 23, p. 145.) If $A$ denotes the graph of $f$; $g:[0,1] \rightarrow L^{1}(\lambda)$ is the identically 0 function, and $h_{1}\left(\omega, \omega^{\prime}\right)=\chi_{A}\left(\omega, \omega^{\prime}\right)$ for $\left(\omega, \omega^{\prime}\right) \in[0,1] \times[0,1]$, then for each choice of $\omega, h_{1}(\omega, \cdot)=g(\omega, \cdot)$ 
[a.e. $\lambda$ ] since both are 0 almost everywhere, but $h_{1}$ is not jointly measurable since $A$ is nonmeasurable. Professor P. Erdös kindly pointed out this example to us. Thus the proof of the proposition is complete, and consequently that of Theorem 2.1 as well.

In fact, the argument presented in the proof of Theorem 2.1 may be slightly modified to prove the following, formally stronger result.

CoRollary 2.3. Assume that $K$ has the RNP. If $\mu_{1}, \mu_{2} \in \mathscr{F}_{t}(K)$ and if $\mu<_{d} \mu_{2}$, then for each $f \in L_{K}^{1}(P)$ such that $f(P)=\mu_{1}$ there is $a g \in L_{K}^{1}(P)$ with $g(P)=\mu_{2}$ and $f<_{m} g$.

Proof. It is straightforward to check that if $f \in L_{K}^{1}(P)$ then there is a countable ordinal $\beta$ such that $f \in \mathscr{F}_{\beta}$. Throughout the proof of Theorem 2.1 the first $\bar{\Omega}$-factor of $\Omega$ may be replaced by $\{0,1\}^{\beta}$ with only notational changes necessary in the subsequent proofs. (The second $\bar{\Omega}$-factor used, for example in the definition of $\bar{g}_{1}$, need not be alternated.) Proposition 2.2 must also be modified appropriately, but the changes here too are cosmetic only. Indeed, nowhere in the proof of the proposition is it important that the first and second factors be identical, nor that the first factor be a separable probability space.

Another way of looking at 2.1 and 2.3 is to introduce an equivalence relation $\sim$ on $L_{K}^{1}(P)$ so that $f \sim g$ if and only if $f(P)=g(P)$. Let $[f]=\left\{g \in L_{K}^{1}(P): f \sim g\right\}$ and let $\mathscr{L}=\left\{[f]: f \in L_{K}^{1}(P)\right\}$. Define $I: \mathscr{L} \rightarrow \mathscr{P}_{t}(K)$ by $I([f])=f(P)$. Then $I$ is $1-1$ and onto. The proof of the following result is immediate from Theorem 2.1.

THEOREM 2.4. Suppose that $K$ has the RNP. Write $[f]<[g]$ if there are $f_{1} \in[f]$ and $g_{1} \in[g]$ such that $f_{1}<_{m} g_{1}$. Then $I$ is an order isomorphism between $(\mathscr{L},<)$ and $\left(\mathscr{P}_{t}(K),<_{d}\right)$. (In particular, $<$ is a partial order on $\mathscr{L}$.)

Our next result provides information about the structure of $[f]$ for $f \in L_{K}^{1}(P)$.

THeOREM 2.5. Let $f$ and $g$ be functions in $L_{K}^{1}(P)$. If $f<_{m} g$ and $f \neq g$ on a set of positive measure then $f(P) \neq g(P)$. That is, no two elements of $[f]$ are commensurable.

N. B. The RNP assumption is not needed.

Proof. It is convenient to consider the case $f=E\left[g \mid \mathscr{F}_{\xi}\right]$ where $\xi$ is the first infinite ordinal since notation has already been developed 
earlier for dealing with this situation. The general case $\left(f=\boldsymbol{E}\left[g \mid \mathscr{F}_{\beta}\right]\right.$ for some $\beta<\alpha$ ) differs from this one notationally only. By modifying $f$ and $g$ on sets of measure 0 , assume that each is separablyvalued with all values in $K$, and that $g$ is $\mathscr{B}(\Omega)$-measurable. The function $g$ is not $\mathscr{F}_{\xi}$-measurable since $f$ and $g$ differ on a set of positive measure by assumption. As in the proof of Theorem 2.1, we will sometimes write $\Omega=\bar{\Omega} \times \Omega_{1}, P=\bar{P} \times P_{1}$ and $\omega=\left(\omega_{\xi}, \omega^{\prime}\right)$ (where it is understood that $\omega_{\xi} \in \bar{\Omega}$ and $\omega_{1} \in \Omega_{1}$ ).

Observe that there is a set $\bar{B} \in \mathscr{B}(\bar{\Omega})$ with $\bar{P}(\bar{B})>0$ such that whenever $\omega_{\xi} \in \bar{B}$ then the function $g\left(\omega_{\xi}, \cdot\right): \Omega_{1} \rightarrow K$ is not [a.e. $P_{1}$ ] a constant function. Indeed, assume the contrary. Pick a set $\bar{A}$ of $\bar{P}$-measure 1 such that $g\left(\omega_{\xi}, \cdot\right)$ is almost everywhere constant for each choice of $\omega_{\xi} \in \bar{A}$. Let $h\left(\omega_{\xi}\right)=\int_{\Omega_{1}} g\left(\omega_{\xi}, \omega^{\prime}\right) d P_{1}\left(\omega^{\prime}\right)$ for each $\omega_{\xi} \in \bar{\Omega}$. Since $g$ is $\mathscr{B}(\Omega)$-measurable (hence $\mathscr{B}(\bar{\Omega}) \times \mathscr{B}\left(\Omega_{1}\right)$-measurable) the Fubini theorem asserts that $h$ is $\mathscr{B}(\bar{\Omega})$-measurable. Clearly $h\left(\omega_{\xi}\right)$ is the value that $g\left(\omega_{\xi}, \cdot\right)$ assumes [a.e. $P_{1}$ ] for $\omega_{\xi}$ in $\bar{A}$. Let $C=$ $\left\{\left(\omega_{\xi}, \omega^{\prime}\right) \in \Omega: h\left(\omega_{\xi}\right) \neq g\left(\omega_{\xi}, \omega^{\prime}\right)\right\}$. Then $C \in \mathscr{B}(\Omega)$ and

$$
\int_{\bar{\Omega}} \chi_{C} d P=\int_{\bar{A}} \int_{\bar{\Omega}_{1}} \chi_{C}\left(\omega_{\xi}, \omega^{\prime}\right) d P_{1}\left(\omega^{\prime}\right) d \bar{P}\left(\omega_{\xi}\right)=0 .
$$

Consequently $g$ is $\mathscr{F}_{\xi}$-measurable (since it differs from $h$ on a set of measure 0$)$. This impossibility shows that $g\left(\omega_{\xi}, \cdot\right)$ is not [a.e. $P_{1}$ ] constant for $\omega_{\xi}$ in some set $\bar{B} \in \mathscr{B}(\bar{\Omega})$ of positive measure, as associated above.

Since $f$ and $g$ have separable ranges, $R_{f}$ and $R_{g}$, there is a convex function $F \in C_{b}(K)$ which is strictly convex on the closed convex hull of $R_{f} \cup R_{g}$. (For example, choose $\left\{F_{i}\right\}_{i=1}^{\infty} \subset E^{*}$ such that $\left\|F_{i}\right\|=1$ for each $i$ and $\left\{F_{i}\right\}_{i=1}^{\infty}$ separates the points of the closed convex hull of $R_{f} \cup R_{g}$, and let $F=\sum_{i=1}^{\infty} 1 / 2^{i} F_{i}^{2}$.) Moreover, one version of $E\left[g \mid \mathscr{F}_{\xi}\right]$ is given by the formula

$$
E\left[g \mid \mathscr{F}_{\xi}\right]\left(\omega_{\xi}, \omega_{1}\right)=\int_{\Omega_{1}} g\left(\omega_{\xi}, \omega^{\prime}\right) d P_{1}\left(\omega^{\prime}\right)
$$

(To see this, note first that the function $\left(\omega_{\xi}, \omega_{1}\right) \rightarrow \int_{\Omega_{1}} g\left(\omega_{\xi}, \omega^{\prime}\right) d P_{1}\left(\omega^{\prime}\right)$ is $\mathscr{B}(\bar{\Omega}) \times\left\{\Phi, \Omega_{1}\right\}$-measurable by Fubini's theorem. If $D \in \mathscr{F}_{\xi}$, it differs from some set $\bar{D} \times \Omega_{1}$ (where $\bar{D} \in \mathscr{B}(\bar{\Omega})$ ) on a set of measure 0 , and hence

$$
\begin{aligned}
\int_{D} \int_{\Omega_{1}} g\left(\omega_{\xi}, \omega^{\prime}\right) d P_{1}\left(\omega^{\prime}\right) d P(\omega) & =\int_{\bar{D} \times \Omega_{1}} g\left(\omega_{\xi}, \omega^{\prime}\right) d \bar{P} \times P_{1}\left(\omega_{\xi}, \omega^{\prime}\right) \\
& =\int_{D} g d P
\end{aligned}
$$

Thus (**) holds.) Finally, note that if $\omega_{\xi} \in \bar{B}$ then there is a set 
$A \in \mathscr{B}\left(\Omega_{1}\right)$ such that $0<P_{1}(A)<1$ and

$$
\frac{1}{P_{1}(A)} \int_{A} g\left(\omega_{\hat{\xi}}, \omega^{\prime}\right) d P_{1}\left(\omega^{\prime}\right) \neq \frac{1}{1-P_{1}(A)} \int_{\Omega_{1} \backslash A} g\left(\omega_{\xi}, \omega^{\prime}\right) d P_{1}\left(\omega^{\prime}\right)
$$

Thus, by the strict convexity of $F$ on the closed convex hull of $R_{f} \cup R_{g}$, as well as by Jensen's inequality [15], we have

$$
\begin{aligned}
& F\left(\int_{\Omega_{1}} g\left(\omega_{\xi}, \omega^{\prime}\right) d P_{1}\left(\omega^{\prime}\right)\right) \\
& \quad<P_{1}(A) F\left(\int_{A} g d \frac{P_{1}}{P_{1}(A)}\right)+\left(1-P_{1}(A)\right) F\left(\int_{\Omega_{1} \backslash A} g d \frac{P_{1}}{1-P_{1}(A)}\right) \\
& \left.\quad \leqq P_{1}(A) \int_{A} F \circ g d \frac{P_{1}}{P_{1}(A)}+\left(1-P_{1}(A)\right) \int_{\Omega_{1} \backslash A} F \circ g d \frac{P_{1}}{1-P_{1}(A)}\right) \\
& \quad=\int_{\Omega_{1}} F \circ g\left(\omega_{\xi}, \omega^{\prime}\right) d P_{1}\left(\omega^{\prime}\right) .
\end{aligned}
$$

Consequently

$$
\begin{aligned}
\int_{K} F d f(P) & =\int_{\Omega} F \circ f(\omega) d P(\omega)=\int_{\Omega} F \circ E\left[g \mid \mathscr{F}_{\xi}\right](\omega) d P(\omega) \\
& =\int_{\bar{\Omega}} F\left(\int_{\Omega_{1}} g\left(\omega_{\xi}, \omega^{\prime}\right) d P_{1}\left(\omega^{\prime}\right)\right) d \bar{P}\left(\omega_{\xi}\right) \\
& =\int_{\bar{\beta}} F\left(\int_{\Omega_{1}} g d P_{1}\right) d \bar{P}+\int_{\bar{\Omega} \bar{B}} F\left(\int_{\Omega_{1}} g d P_{1}\right) d \bar{P} \\
& <\int_{\bar{B}} \int_{\Omega_{1}} F \circ g d P_{1} d \bar{P}+\int_{\bar{\Omega} \backslash \bar{B}} F\left(\int_{\Omega_{1}} g d P_{1}\right) d \bar{P} \\
& \leqq \int_{\bar{\Omega}} \int_{\Omega_{1}} F \circ g d P_{1} d \bar{P}=\int_{\Omega} F \circ g d P=\int_{K} F d g(P) .
\end{aligned}
$$

Thus $f(P) \neq g(P)$ as was to be shown.

In [13] Mankiewicz showed that when $K$ has the RNP, $\mu \in \mathscr{P}_{t}(K)$ is separable extremal if and only if $\mu$ is $<_{d}$-maximal. (See [13] or [2] for definitions and proofs.) The corresponding result concerning the partially ordered sets $\left(L_{K}^{1}(P),<_{m}\right)$ and $\left(\mathscr{P}_{t}(K),<_{d}\right)$ is a consequence of Theorem 2.5.

Corollary 2.6. Assume that $K$ has the RNP. Then $f \in L_{K}^{1}(P)$ is $<_{m}$-maximal if and only if $f(P)$ is $<_{d}$-maximal.

Proof. Mankiewicz [13] proved that if $f$ is $<_{m}$-maximal then $f(P)$ is separable extremal and hence $<_{d}$-maximal. (Alternatively, see [2].) Conversely, suppose that $\mu \in \mathscr{P}_{t}(K)$ is $<_{d}$-maximal and that $f(P)=\mu$. If $f<_{m} g$ for some $g \in L_{K}^{1}(P)$ then $f(P)<_{m} g(P)$ (Theorem 2.1) and consequently $f(P)=g(P)$. It follows from Theorem 2.5 that $f=g$ [a.e. $P$ ]. That is, $f$ is $<_{m}$-maximal. 
Other orderings are suggested by the setup used in Corollary 2.4. Suppose that $\mathscr{C}$ is a refinement of $\mathscr{L}$. (That is, $\mathscr{C}$ is a partition of $L_{K}^{1}(P)$ into pairwise disjoint sets, each of which is a subset of some $[f]$ for some $f \in L_{K}^{1}(P)$.) If $M, N \in \mathscr{l l}$, write $M \ll N$ if there are $f \in M$ and $g \in N$ such that $f<_{m} g$. (For example, let $\Psi=\{\psi: \alpha \rightarrow \alpha: \psi$ is $1-1$ and onto $\}$ denote the collection of $1-1$ correspondences on the countable ordinals. When $\psi \in \Psi$ and $\omega \in \Omega$ let $\psi(\omega)$ be that point of $\Omega$ whose $\beta$ th coordinate $(\beta<\alpha)$ is $\omega(\psi(\beta))$. Furthermore, for $f \in L_{K}^{1}(P)$ let $M_{f}=\left\{g \in L_{K}^{1}(P)\right.$ : there is a. $\psi \in \Psi$ such that $f \circ \psi=g\}$. Note that if $f_{1} \in M_{f}$ - so that $f_{1}=f \circ \psi$ for some $\psi \in \Psi$ - then $f(P)=f_{1}(P)$ and hence $f_{1} \in[f]$. Moreover, $\left\{M_{f}: f \in L_{K}^{1}(P)\right\}$ clearly partitions $L_{K}^{1}(P)$ into pairwise disjoint sets. As above, we write $M_{f} \ll M_{g}$ if there are $f_{1} \in M_{f}$ and $g_{1} \in M_{g}$ such that $f_{1}<_{m} g_{1}$.)

THEOREM 2.7. «is a reflexive and antisymmetric order. If $K$ has the RNP then the following are equivalent:

(1) $M \in \mathscr{C l}$ is «-maximal.

(2) $f$ is $<_{m}$-maximal for each $f \in M$.

(3) $f$ is $<_{m}$-maximal for some $f \in M$.

(4) $f(P)$ is $<_{d}$-maximal for each (some) $f \in M$.

Proof. Reflexivity is (self) evident. Suppose now that $L \ll M$ and $M \ll L$. Then there are functions $f_{1}, f_{2} \in L$ and $g_{1}, g_{2} \in M$ such that $f_{1}<_{m} g_{1}$ and $g_{2}<_{m} f_{2}$. Let $f_{1}(P)=\mu_{1}$ and $g_{1}(P)=\mu_{2}$. Since $\mathscr{C l}$ is a refinement of $\mathscr{L}$ it follows that $f_{2} \in\left[f_{1}\right]$ and $g_{2} \in\left[g_{1}\right] . \quad$ By Theorem 2.1,

$$
f_{1}(P)=\mu_{1}<_{d} g_{1}(P)=\mu_{2}=g_{2}(P)<_{d} f_{2}(P)=\mu_{1} .
$$

Consequently $\mu_{1}=\mu_{2}$ (since $<_{d}$ is a partial order) and hence $g_{1} \in\left[f_{1}\right]$. But then Theorem 2.5 applies. Thus $f_{1}=g_{1}$ [a.e. $P$ ] and hence $f_{1} \in$ $L \cap M$. Thus $L=M$.

The equivalence of statements (2), (3), and (4) was established in Corollary 2.6. Suppose next that $M$ is $\ll$-maximal and that $f \in M$. If $f<_{m} g$ and $f$ and $g$ differ on a set of positive $P$-measure then $f(P) \neq g(P)$ by Theorem 2.5. Let $g \in N \in \mathscr{Z}$. Then $N \neq M$ since $M \subseteq[f]$ and $N \subseteq[g]$. Also $M \ll N$ by definition. Thus (1) implies (2). Finally, assume that for each $f \in M, f$ is $<_{m}$-maximal. If $M \ll N$ then there are $f_{1} \in M$ and $g_{1} \in N$ such that $f_{1}<_{m} g_{1}$. Consequently $f_{1}=g_{1}$ [a.e. $P$ ] and thus $M=N$. Therefore (2) implies (1), and the proof is complete.

It is not difficult to construct examples of partitions $\mathscr{C}$ for which $\ll$ is not transitive. Nevertheless our last result is typical of those for a collection of 'natural' partitions $\mathscr{A}$. 
Proposition 2.8. Let $\mathscr{t}$ be the partition described prior to Theorem 2.7 (in which a typical $M_{f} \in \mathscr{C}$ is of the form $\left\{g \in L_{K}^{1}(P)\right.$ : $g=f \circ \psi$ for some $1-1$ correspondence $\psi$ on the countable ordinals $\}$ ). Then « is a partial order.

N. B. The RNP is not required for this result.

Proof. It suffices, by virtue of Theorem 2.7, to establish the transitivity of $\ll$. For a nonempty set $B$ of countable ordinals (possibly of the form $\beta=U\{\gamma: \gamma<\beta\}$ for $\beta<\alpha$ ) let $\mathscr{B}_{B}$ denote the $\sigma$-algebra of subsets of $\Omega$ generated by $\{\{\omega \in \Omega: \omega(\beta)=0\}: \beta \in B\}$. Write $f_{\psi}$ in place of $f \circ \psi$ and observe that if $f$ is $\mathscr{B}_{B}$-measurable then $f_{\psi}$ is $\mathscr{B}_{\psi-1}$-measurable. Moreover, if $f$ is $\mathscr{B}_{\beta}$-measurable for some $\beta<\alpha$ and if $\omega$ and $\omega^{\prime}$ are points of $\Omega$ for which $\omega(\gamma)=\omega^{\prime}(\gamma)$ for $\gamma<\beta$ then $f(\omega)=f\left(\omega^{\prime}\right)$. For $B$ as above let $\Omega_{B}=\{0,1\}^{B}$ and let $P_{B}$ be the (by now) natural product probability measure on $\mathscr{P}\left(\Omega_{B}\right)$.

Suppose that $M_{f} \ll M_{g}$ and that $M_{g} \ll M_{h}$. In this paragraph we construct functions $f_{1} \in M_{f}, g_{1}, g_{2} \in M_{g}$, and $h_{2} \in M_{h}$, as well as a countable ordinal $\beta$, such that

(a) $f_{1}<_{m} g_{1}, g_{2}<_{m} h_{2}$

(b) $f_{1}$ and $g_{1}$ are $\mathscr{B}_{\beta}$-measurable; $g_{2}$ and $h_{2}$ are $\mathscr{B}_{B}$-measurable where $B=\{\gamma: \beta+1 \leqq \gamma \leqq 2 \beta\}$. Indeed, if $f_{1} \in M_{f}, g_{1}, g^{\prime} \in M_{g}$ and $h^{\prime} \in M_{h}$ are chosen so that $f_{1}<_{m} g_{1}$ and $g^{\prime}<_{m} h^{\prime}$, each may be modified on a set of measure 0 so that they become $\mathscr{B}(\Omega)$-measurable. Since $\mathscr{B}(\Omega)=\bigcup_{\beta<\alpha} \mathscr{B} \beta$ it is possible to choose $\beta<\alpha$ such that $f_{1}, g_{1}, g^{\prime}$ and $h^{\prime}$ are $\mathscr{B}_{\beta}$-measurable. Choose any $\psi \in \Psi$ such that $\psi(\beta+\gamma)=\gamma$ for each $\gamma$ in the interval $1 \leqq \gamma \leqq \beta$. We will show that $g_{\psi}^{\prime}<_{m} h_{\psi}^{\prime}$. (Once this is established, conditions (a) and (b) will be met by $\beta, f_{1}$, $g_{1}, g_{2}=g_{\psi}^{\prime}$, and $h_{2}=h_{\psi}^{\prime}$.) Pick $\eta \leqq \beta$ such that $g^{\prime}=E\left[h^{\prime} \mid \mathscr{B}_{\eta}\right]$. Then $g_{\psi}^{\prime}$ is $\mathscr{B}_{\beta+\eta}$-measurable. In order to prove that $E\left[h_{\psi}^{\prime} \mid \mathscr{B}_{\beta+\eta}\right]=g_{\psi}^{\prime}$ it thus suffices to demonstrate that $\int_{A} h_{\psi}^{\prime} d P=\int_{A} g_{\psi}^{\prime} d P$ for each $A \in \mathscr{B}_{\beta+\eta}$. Let $C=\{\gamma<\alpha: \beta+\eta \leqq \gamma\}$, and choose $A_{1} \in \mathscr{B}\left(\Omega_{\beta}\right)$ and $A_{2} \in \mathscr{B}\left(\Omega_{\{\beta+\gamma: r<\eta\}}\right)$. Then $h^{\prime}$ does not depend on the $\psi(C)$ coordinates (since they are each past $\beta$ ). Thus

$$
\begin{aligned}
& \int_{A_{1} \times A_{2} \times \Omega_{C}} h_{\psi}^{\prime} d P=\int_{\psi\left(A_{1}\right) \times \psi\left(A_{2}\right) \times \Omega \psi(C)} h^{\prime} d P \\
& =\int_{\psi\left(A_{1}\right)} \int_{\psi\left(A_{2}\right)} \int_{\Omega \psi_{(C)}} h^{\prime} d P_{\psi(C)} d P_{\psi(\langle\beta+\gamma: i<\eta)} d P_{\psi(B)} \\
& =\int_{\psi\left(A_{1}\right)} \int_{\psi\left(A_{2}\right)} h^{\prime} d P_{\gamma_{1}} d P_{\psi(B)} \text {. }
\end{aligned}
$$

But $\psi\left(A_{2}\right) \in \mathscr{B}_{\eta}$ and since $E\left[h^{\prime} \mid \mathscr{B}_{\eta}\right]=g^{\prime}$ the above calculation may 
be continued as follows:

$$
\begin{aligned}
\int_{A_{1} \times A_{2} \times \Omega_{C}} h_{\psi}^{\prime} d P & =\int_{\psi\left(A_{1}\right)} \int_{\psi\left(A_{2}\right)} g^{\prime} d P_{\eta} d P_{\psi(B)} \\
& =\int_{A_{1} \times A_{2} \times \Omega_{C}} g_{\psi}^{\prime} d P .
\end{aligned}
$$

Thus $\int_{A} h_{\psi}^{\prime} d P=\int_{A} g_{\psi}^{\prime} d P$ where $A=A_{1} \times A_{2} \times \Omega_{C}$. The same equation therefore obtains more generally for all finite disjoint unions of sets of the form $A_{1} \times A_{2} \times \Omega_{C}$. This collection of sets is an algebra which generates $\mathscr{B}_{\beta+r}$, and a standard argument shows that the above equation extends to all $A \in \mathscr{B}_{\beta+\eta}$ as desired. (Recall: $g_{\psi}^{\prime}$ and $h_{\psi}^{\prime}$ take their values in the bounded set $K$.)

In the new notation, then, $E\left[h_{2} \mid \mathscr{B}_{\beta+\gamma}\right]=g_{2}$. Define $\psi^{\prime}$ as follows: $\psi^{\prime} \gamma^{\prime}(2 \beta+\gamma)=\beta+\gamma$ and $\psi^{\prime}(\beta+\gamma)=2 \beta+\gamma$, for $\eta \leqq \gamma<\beta$, while $\gamma^{\prime}(\gamma)=\gamma$ otherwise for $\gamma<\alpha$. Let $h_{3}=\left(h_{2}\right)_{\psi \prime}$. It is easy to check that $\boldsymbol{E}\left[h_{3} \mid \mathscr{B}_{\beta+\eta}\right]=g_{2}$. Now find $\varphi \in \Psi$ such that $\left(g_{2}\right)_{\varphi}=g_{1}$ and pick $\varphi^{\prime} \in \Psi$ such that

(1) $\varphi^{\prime-1}(\gamma)<\beta+\eta$ whenever $\gamma<\beta+\eta$.

(2) $\varphi^{\prime}(\gamma)=\varphi(\gamma)$ if both $1 \leqq \gamma<\beta$ and $\beta \leqq \varphi(\gamma)<\beta+\eta$. It follows from $(2)$ that $\left(g_{2}\right)_{\varphi}=\left(g_{2}\right)_{\varphi}=g_{1}$.

Since $g_{1}$ is $\mathscr{B}_{\beta}$-measurable it is $\mathscr{B}_{\beta+\gamma}$-measurable. It is a consequence of (1) that if $A_{1} \in \mathscr{B}\left(\Omega_{\beta+\eta}\right)$ then $\varphi^{\prime}\left(A_{1} \times \Omega_{C}\right) \in \mathscr{B}_{\beta+\eta}$. Thus, for such sets $A_{1}$ we have

$$
\begin{aligned}
\int_{A_{1} \times \Omega_{C}}\left(h_{3}\right)_{\varphi^{\prime}} d P & =\int_{\varphi^{\prime}\left(A_{1}\right) \times \Omega_{\varphi^{\prime}(C)}} h_{3} d P \\
& =\int_{\varphi^{\prime}\left(A_{1}\right) \times \Omega_{\varphi^{\prime}}(C)} g_{2} d P \\
& =\int_{A_{1} \times \Omega_{C}}\left(g_{2}\right)_{\varphi} d P=\int_{A_{1} \times \Omega_{C}} g_{1} d P .
\end{aligned}
$$

Thus $\boldsymbol{E}\left[\left(h_{3}\right)_{\varphi} \mid \mathscr{B}_{\beta+r}\right]=g_{1}$. That is, $f_{1}<_{m} g_{1}<_{m}\left(h_{3}\right)_{\varphi}$. Consequently $f_{1}<\left(h_{3}\right)_{\varphi}$ and thus

$$
M_{f}=M_{f_{1}} \ll M_{\left(h_{3}\right) \varphi^{\prime}}=M_{h}
$$

as was to be shown.

\section{REFERENCES}

1. E. M. Alfsen, Compact Convex Sets and Boundary Integrals, Ergebnisse der Mathematik und Ihrer Grenzgebiete, Band 57, Springer-Verlag, Berlin, 1971.

2. R. D. Bourgin, Geometric aspects of Banach spaces with the Radon-Nikodym property, (in progress).

3. R. D. Bourgin and G. A. Edgar, Noncompact simplexes in Banach spaces with the Radon-Nikodým property, J. Functional Analysis, 23 No. 2 (1976), 162-176. 
4. G. Choquet, Lectures on Analysis, Vol. 2, Mathematics Lecture Note Series, W. A. Benjamin, Inc., 1969.

5. J. Diestel, Geometry of Banach Spaces-Selected Topics, Vol. 485, Lecture Notes in Mathematics, Springer-Verlag, Heidelberg, 1975.

6. R. M. Dudley, Convergences of Baire measures, Studia Math., 27 (1966), 251-268.

7. G. A. Edgar, Extremal integral representations, J. Functional Analysis, 23 No. 2 (1976), 145-161.

8. - On the Radon-Nikodym property and martingale convergence, Proceedings of the Conference on Vector Space Measures and Applications, Vol. 2. To appear.

9. E. Hille, Methods in Classical and Functional Analysis, Addison-Wesley Series in Mathematics, Addison-Welsley, Reading, Mass., 1972.

10. E. Hille and R. S. Phillips, Functional Analysis and Semigroups, Vol. XXXI, Amer. Math. Soc. Colloquium Publications, Amer. Math. Soc., Providence, 1957.

11. K. Kuratowski and C. Ryll-Nardzewski, A general theorem on selectors, Bull. Acad. Polon. Sci., 13 (1965), 397-402.

12. L. LeCam, Convergence in distribution of stochastic processes, Univ. of Calif. Publ. in Stat., 2 (1957), 207-236.

13. P. Mankiewicz, A remark on Edgar's extremal integral representation theorem, to appear in Studia Math.

14. E. Marczewski and R. Sikorski, Measures in nonseparable metric spaces, Colloq. Math., 1 (1948), 133-139.

15. P. A. Meyer, Probability and Potentials, Blaisdell, Waltham, Mass., 1966.

16. J. C. Oxtoby and S. Ulam, On the existence of a measure invariant under a transformation, Ann. of Math., (2) 40 (1939), 560-566.

17. R. R. Phelps, Lectures on Choquet's Theorem, Van Nostrand Mathematical Studies No. 7, Van Nostrand, Princeton, 1966.

18. F. S. Scalora, Abstract martingale convergence theorems, Pacific J. Math., 11 (1961), 347-374.

19. R. M. Solovay, Real-valued Measurable Cardinals, in Axiomatic Set Theory, (D. S. Scott, Ed.), Amer. Math. Soc., Providence, R. I., 1967, 397-428.

20. J. Saint Raymond, Représentation Intégrale dans Certains Convexes, Séminaire Choquet (14e année: 1974/75), Initiation à l'analyse, Exp. No. 2, Paris, 1975.

Received March 20, 1978.

Sabino, New Mexico 


\section{PACIFIC JOURNAL OF MATHEMATICS}

EDITORS

DONALD BABBITT (Managing Editor)

University of California

Los Angeles, California 90024

HUGo RossI

University of Utah

Salt Lake City, UT 84112

C. C. MOORE

J. DUGUNDJI

Department of Mathematics

University of Southern California

Los Angeles, California 90007

R. FinN AND J. MiLgraM

Stanford University

Stanford, California 94305

University of California

Berkeley, CA 94720

\section{ASSOCIATE EDITORS}

E. F. BrCKENBACH

B. H. NeUmanN

F. WOLF

K. YoshidA

\section{SUPPORTING INSTITUTIONS}

UNIVERSITY OF BRITISH COLUMBIA

UNIVERSITY OF SOUTHERN CALIFORNIA

CALIFORNIA INSTITUTE OF TECHNOLOGY

STANFORD UNIVERSITY

UNIVERSITY OF CALIFORNIA

UNIVERSITY OF HAWAII

MONTANA STATE UNIVERSITY

UNIVERSITY OF TOKYO

UNIVERSITY OF NEVADA, RENO

UNIVERSITY OF UTAH

NEW MEXICO STATE UNIVERSITY

WASHINGTON STATE UNIVERSITY

OREGON STATE UNIVERSITY

UNIVERSITY OF WASHINGTON

UNIVERSITY OF OREGON 


\section{Pacific Journal of Mathematics}

\section{Vol. 81, No. $1 \quad$ November, 1979}

Thomas E. Armstrong, Simplicial subdivision of infinite-dimensional compact cubes ..................................... 1

Herbert Stanley Bear, Jr., Approximate identities and pointwise convergence ................................

Richard David Bourgin, Partial orderings for integral representations on convex sets with the Radon-Nikodým property..................

Alan Day, Herbert S. Gaskill and Werner Poguntke, Distributive lattices

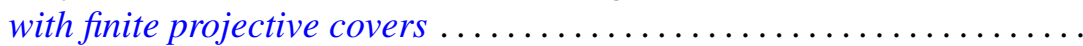

Heneri Amos Murima Dzinotyiweyi and Gerard L. G. Sleijpen, A note on measures on foundation semigroups with weakly compact orbits ......

Ronald James Evans, Resolution of sign ambiguities in Jacobi and Jacobsthal sums ...................................

John Albert Fridy, Tauberian theorems via block dominated matrices ......

Matthew Gould and Helen H. James, Automorphism groups retracting onto

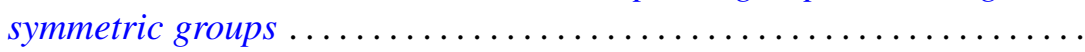

Kurt Kreith, Nonlinear differential equations with monotone solutions . . . . 101

Brian William McEnnis, Shifts on indefinite inner product spaces........ 113

Joseph B. Miles, On entire functions of infinite order with radially

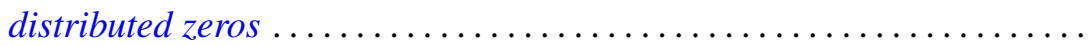

Janet E. Mills, The idempotents of a class of 0-simple inverse semigroups ...............................

Edward Jean Moulis, Jr., Generalizations of the Robertson functions ...

Richard A. Moynihan and Berthold Schweizer, Betweenness relations in probabilistic metric spaces.......................

Stanley Ocken, Perturbing embeddings in codimension two ....

Masilamani Sambandham, On the average number of real zeros of a class of random algebraic curves.

Jerry Searcy and B. Andreas Troesch, A cyclic inequality and a related eigenvalue problem.

Roger R. Smith and Joseph Dinneen Ward, $M$-ideals in $B\left(l_{p}\right)$...

Michel Talagrand, Deux généralisations d'un théorème de I. Namioka ..

Jürgen Voigt, $O n Y$-closed subspaces of $X$, for Banach spaces $X \subset Y$;

existence of alternating elements in subspaces of $C(J)$

Sidney Martin Webster, On mapping an $n$-ball into an $(n+1)$-ball in complex spaces

David J. Winter, Triangulable subalgebras of Lie p-algebras ... 